\title{
UNA NUEVA ESPECIE DE ARBUTUS (ERICACEAE, ARBUTEAE) DE LA SIERRA MADRE OCCIDENTAL, MEXICO
}

\author{
M. Socorro Gonzalez Elizondo' y Martha Gonzalez Elizondo' \\ CIIDIR Unidad Durango \\ Instituto Politécnico Nacional \\ Apartado Postal 738 \\ 34000 Durango, Dgo., México
}

\begin{abstract}
RESUMEN
Se describe como nueva para la ciencia a Arbutus madrensis de los estados de Sinaloa, Durango, Jalisco y Nayarit. La especie en cuestión es un árbol común en bosques de pino y de pino-encino en la Sierra Madre Occidental entre los 2400 y $2800 \mathrm{~m}$ de altitud y se distingue de otras especies de madroño por su corteza rugosa, persistente hasta en las últimas ramillas y por sus hojas grandes, ampliamente ovadas a elípticas, con pubescencia ferruginea no glandular.
\end{abstract}

\begin{abstract}
Arbutus madrensis, from the states of Sinaloa, Durango, Jalisco and Nayarit is described. The new species is a common tree in pine and pine-oak forests of the Sierra Madre Occidental between 2400 and $2800 \mathrm{~m}$. It can be distinguished from other madrones by its checkered and persistent bark present even on the last twigs, and the large, widely ovate to elliptic leaves with ferruginous, non glandular pubescence.
\end{abstract}

Las especies del género Arbutus son un importante componente de los bosques templados de México, tanto por su abundancia como por su amplia distribución. Los madroños, como se conoce comunmente a estas plantas, forman parte de los bosques de pino y de pino-encino, y ocasionalmente llegan a constituir masas puras, pero su taxonomía ha sido confusa.

Desde 1924, en que Standley sugiriera la posibilidad de que todas las plantas mexicanas del género debieran ser consideradas como meras formas de una especie variable (A. xalapensis), hasta fechas muy recientes, son varios los trabajos en que se vierte la opinión de que el grupo presenta gran diversidad morfologica y caracteres poco constantes (McVaugh \& Rosatti, 1978; Sorensen, 1987; González Villarreal, 1990).

El primer avance importante en el conocimiento del grupo después del trabajo de Small (1914), fue la cautelosa descripción de A. occidentalis por McVaugh y Rosatti en 1978. Otra especie más, A. tessellata, fue descrita por Sorensen en 1987.

\footnotetext{
1 Becarias de la COFAA del Instituto Politécnico Nacional.
} 
Después de la revisión del material depositado en los herbarios CIIDIR, ENCB, IEB, MEXU y NMU, y del muestreo de poblaciones en la Sierra Madre Occidental, González Elizondo y González Elizondo (en prensa) reconocieron seis especies y dos variedades de Arbutus para dicha área, considerando como nuevo para la ciencia a uno de dichos taxa, el cual se describe formalmente en este trabajo:

\section{Arbutus madrensis S. González-Elizondo, sp. nov.}

Arbor sempervirens, 2-9 (-15) m alta, cortex tessellata; folia late ovata vel elliptica, $15 \mathrm{~cm}$ longa vel breviora, integerrima, raro dentata, supra atroviridia, laevigata, subtus ferrugineo-pubescentia, apice obtusa vel acuta, basi obtusa vel subcordata; petioli $2-3 \mathrm{~cm}$ longi; inflorescentiae terminales et subterminales, multiracemosae, quoque racemo $2-7 \mathrm{~cm}$ longo; pedicellis bracteolatis, bracteis $3.5-5 \mathrm{~mm}$ longis; flores luteoli, calyx ca. $1.6 \mathrm{~mm}$ longus, lobis 5 , corolla angulato-urceolata, $4-8 \mathrm{~mm}$ longa, usque ad $6 \mathrm{~mm}$ diametro, stamina 10 , filamentis 1.5-2.2 mm longis, ovarium pilosum, 5-loculare, ovula quoque loculo ca. 6 8; fructus depresse globosus, papillosus, aurantiacus, usque ad $12 \mathrm{~mm}$ diametro, ca. 10spermus, seminibus 2-2.8 $\mathrm{mm}$ longis, 1-1.5 mm latis. (Fig. 1).

Arbol perennifolio de 2 a $9(-15) \mathrm{m}$ de alto. Corteza de color gris oscuro, rugosa y persistente en placas hasta en las últimas ramillas, con excepción de las partes en crecimiento activo. Hojas ampliamente ovadas a elipticas, de 8 a $15 \mathrm{~cm}$ de largo por 6 a $9 \mathrm{~cm}$ de ancho, con el ápice obtuso o a veces agudo, la base redondeada a subcordada, el margen plano, entero, rara vez dentado; haz de color verde oscuro, brillante, glabro o con escasa pubescencia pronto decidua, envés de color verde pálido, opaco, con pubescencia ferrugínea concentrada principalmente hacia la base y sobre la prominente nervadura central; pecíolo de 2 a $3 \mathrm{~cm}$ de largo, con pubescencia blanca a ferrugínea. Inflorescencias terminales y subterminales, pseudopaniculadas, compuestas de varios racimos de 2 a $7 \mathrm{~cm}$ de largo, compactos antes de la antesis, alargándose en la fructificación, ejes y pedicelos cubiertos con corta pubescencia glandular, pelos de 0.2 a $0.3(-0.7) \mathrm{mm}$ de longitud; pedicelos de hasta $1 \mathrm{~cm}$ de largo, engrosados hacia el ápice en la madurez, con una bráctea basal de 3.5 a $5 \mathrm{~mm}$ de largo, navicular, glandular pubescente en la cara abaxial, glabra y brillante en la adaxial, cubriendo dos bractéolas opuestas, elíptico lanceoladas, de hasta $2.6 \mathrm{~mm}$ de longitud; flores escasamente aromáticas; cáliz con lóbulos libres, ampliamente triangulares a deltoideos, hasta de 1.6 $\mathrm{mm}$ de largo por 1.7 a $2 \mathrm{~mm}$ de ancho, reflejos y persistentes en el fruto; corola de color amarillento o amarillo verdoso, anguloso urceolada, de (4-) 6 a $8 \mathrm{~mm}$ de largo por 4 a $6 \mathrm{~mm}$ de diámetro, glabra o con 5 hileras longitudinales de pelos cortos en la parte interna, lóbulos 5, de $1 / 40$ menos del largo total de la corola, hasta de $1.8 \mathrm{~mm}$ de ancho, subreniformes, con márgenes auriculados imbricados; estambres 10, insertos en la base de la corola, bajo el disco nectarífero, filamentos de 1.5 a $2.2 \mathrm{~mm}$ de largo incluyendo la mitad basal conspicuamente ensanchada y densamente pubescente, anteras de 1.5 a $1.7 \mathrm{~mm}$ de largo, abriéndose por dos poros apicales oblicuos, alargados, y provistas de dos apéndices reflejos de la mitad o más del largo de la antera; ovario globoso, piloso, pentalocular, cada lóculo con alrededor de 6 a 8 óvulos, estilo de $3 \mathrm{~mm}$ de largo, estigma globoso, papiloso; fruto una baya subglobosa, ligeramente deprimida, hasta de $1 \mathrm{~cm}$ de 


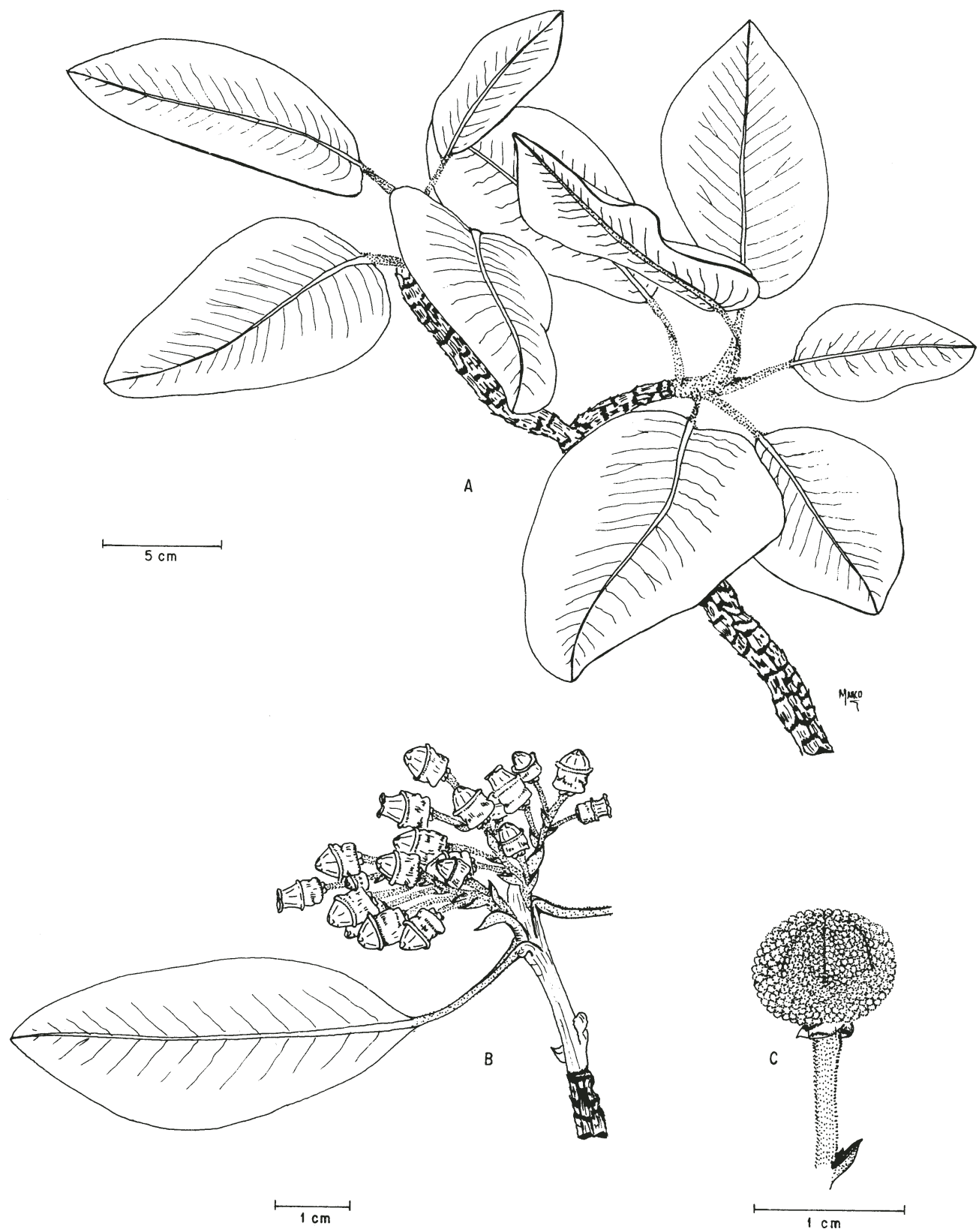

Fig. 1. Arbutus madrensis. A. Aspecto general de hojas y ramillas; B. Inflorescencia; C. Fruto con pedúnculo, bráctea y bractéolas basales 
largo por $1.2 \mathrm{~cm}$ de diámetro, de color amarillo a anaranjado, con la superficie cubierta de papilas altas y carnosas, endocarpio carnoso, blando; semillas alrededor de 10, de color amarillento café, ampliamente fusiformes, de 2 a $2.8 \mathrm{~mm}$ de largo por 1 a $1.5 \mathrm{~mm}$ de ancho, ornamentadas con finas estrías longitudinales, debido al alargamiento de las células de la testa.

TIPO: México, Durango, Las Bayas, $95 \mathrm{~km}$ al $\mathrm{S}$ de la $\mathrm{Cd}$. de Durango, municipio de Durango, bosque de Pinus - Quercus, alt. $2650 \mathrm{~m}$, árbol común localmente en cañadita, 15.VII.1990, S. González Elizondo, A. Garcia y S. Acevedo 4577 (Holotipo ENCB. Isotipos por distribuirse a CIIDIR, IEB, MEXU y otros herbarios). Plantas en fruto.

Nombre común: Madroño, madroño roñoso.

Material adicional examinado: SINALOA, Sierra de Chavarría, 1921, "madroño", J. González Ortega 4072 (US). DURANGO, Bajío de Los Arcos, entre El Huacal y Quebrada Honda, municipio de Tepehuanes, alt. 2380 m, 19.X.1990, M. González Elizondo 2491 (CIIDIR); ibid., M. González Elizondo 2493 (CIIDIR); 2 km de La Manga, rumbo al Ranchito Escondido, por donde se desciende a Quebrada Honda, municipio de Tepehuanes, alt. 2200 m, 26.IV.1989, "madroño (roñoso)", A. Benítez y F. Ramos 502 (CIIDIR, CHAP); Paraje Chamacueros, $30 \mathrm{~km}$ al W de Mesa de Navar, municipio de Tepehuanes, alt. 2860 m, 19.IV.1989, A. Paredes, O. Bravo y F. Ramos 395 (CHAP, CIIDIR); Mesa de los Angeles, Jorge Lara, San Carlos, San Dimas, alt. 2480 m, 13.I.1970, A. May Nah 2847 (MEXU); km 160 de carretera Durango-Mazatlán, municipio de Pueblo Nuevo, alt. 2590 m, 2.XI.1990, S. González 4843 (CIIDIR); ibid., árbol 8 a $10 \mathrm{~m}$ de alto, abundante en exposición al W, S. González 4848 (CIIDIR); 68 km de El Salto, por la carretera a Mazatlán, municipio de Pueblo Nuevo, alt. 2430 m, 11.X.1988, S. González 4324 (CIIDIR); km 141 de carretera Durango-Mazatlán, municipio de Pueblo Nuevo, alt. 2540 m, 3.XI.1990, S. González 4874 (CIIDIR); km 137 de carretera Durango-Mazatlán, municipio de Pueblo Nuevo, alt. 2630 m, 3.XI.1990, S. González 4877 (CIIDIR); km 134 de carretera DurangoMazatlán, municipio de Pueblo Nuevo, alt. 2650 m, 3.XI.1990, S. González 4878 (CIIDIR); Tapextle, a $18 \mathrm{~km}$ de la desviación a la Mina San Luis, municipio de Pueblo Nuevo, alt. 2200 m, 5.VII.1982, R. Hernández M. 7647 y P. Tenorio (MEXU); 26 km al W de El Salto, por la carretera a Mazatlán, municipio de Pueblo Nuevo, 17.I.1990, S. González 4437 (CIIDIR); $52.5 \mathrm{~km}$ de Durango, por la carretera a Mazatlán, alt. $2510 \mathrm{~m}, 10.11 \mathrm{l} .1991, \mathrm{~S}$. González 4914, con P. Sorensen, M. Cházaro y R. Fragoso (CIIDIR); Arroyo La Tecolota, Predio Las Bayas, $95 \mathrm{~km}$ al S de Durango, municipio de Durango, alt. $2710 \mathrm{~m}, 14$. VII.1990, S. González 4506 (CIIDIR); 1 km de La Escondida, municipio de El Mezquital, alt. 2750 m, 4.XI.1990, S. González 4896 (CIIDIR); arroyo El Ranchero, al S de la Reserva de la Biosfera La Michilía, municipio de Súchil, alt. 2550 m, 12.VIII.1990, S. González 4744 (CIIDIR); Mesa El Burro, Reserva de la Biosfera La Michilia, municipio de Súchil, alt. 2580 m, 4.XI.1990, S. González 4890 (CIIDIR); 15 km al SW de Piedra Herrada, por el camino a Los Charcos, municipio de El Mezquital, 10.VI.1981, S. y M. González 1704 (CIIDIR, IEB); Reserva de la Biosfera La Michilía, municipio de Súchil, alt. $2450 \mathrm{~m}, 26$. IV.1988, S. González 4269 con L. Jamieson (CIIDIR); ibid., S. González 4272 con L. Jamieson (CIIDIR); $1 \mathrm{~km}$ al $\mathrm{N}$ de residencia de la Reserva de la Biosfera La Michilla, alt. $2450 \mathrm{~m}$, 
9.III.1991, S. González 4912 con P. Sorensen, M. Cházaro y R. Fragoso (CIIDIR); 55 km de Súchil, por el camino a Los Charcos, municipio de Súchil, 9.III.1991, S. González 4913 con $P$. Sorensen, M. Cházaro y $R$. Fragoso (CIIDIR). NAYARIT, arroyo, $1 \mathrm{~km}$ al $S W$ de Santa Teresa del Nayar, municipio de Jesús Marla, alt. 2100 m, 5.VI.1978, C.L. Diaz Luna 9493 (ENCB). JALISCO, Sierra de Buenavista, al S de Mezquitic, municipio de Mezquitic, alt. $3000 \mathrm{~m}, 29.11 \mathrm{l} .1980$, E. Estrada Faudón s.n. (ENCB); Cerro de Tequila, a rugged volcanic cone $13 \mathrm{~km}$ due $S$ of Tequila, $\mathrm{N}$ facing slopes ca. 100-200 m beneath "microondas" tower, alt. 2800-2900 m, 29.XII.1978, brácteas y flores excepcionalmente grandes, $H$. $H$. Ittis, R. Guzmán, S. Carvajal, M. Nee 1002 (MEXU); Cerro Los Picachos, al E de Tenamaxtlán, municipio de Tenamaxtlán, alt. 2100 m, 10.VII.1986, R. Ramírez Delgadillo 360 (IEB); $8 \mathrm{~km}$ al E de Las Iglesias, campo experimental de la Universidad Autónoma de Chapingo, Sierra de Cacoma, municipio de Ayutla, alt. 2050 m, 9.III.1980, L. M. González Villarreal 1759 (IEB).

Arbutus madrensis se reconoce por su corteza no exfoliante, persistente hasta en las últimas ramillas y por sus grandes hojas ovadas a elípticas, de color verde oscuro con pubescencia ferrugínea no glandular. Comparte con $A$. tessellata y con las formas del norte de $A$. arizonica el carácter de corteza persistente, pero se distingue de ambas especies por las caracteristicas dadas en el cuadro 1. Las formas del sur de A. arizonica presentan corteza exfoliante en las últimas ramillas.

Cuadro 1. Principales características distintivas entre Arbutus tessellata, A. arizonica y A. madrensis.

\begin{tabular}{|c|c|c|c|}
\hline CARACTER & A. tessellata & A. arizonica & A. madrensis \\
\hline $\begin{array}{l}\text { Pubescencia en } \\
\text { pecíolos y hojas }\end{array}$ & $\begin{array}{l}\text { pelos glandulares } \\
\text { rectos, largos, } \\
\text { divergentes en } \\
\text { ángulo recto }\end{array}$ & $\begin{array}{l}\text { ausente o de pelos } \\
\text { muy cortos, aplicados, } \\
\text { no glandulares }\end{array}$ & $\begin{array}{l}\text { pelos ferrugíneos } \\
\text { nunca glandulares } \\
\text { ni divergentes en } \\
\text { ángulo recto }\end{array}$ \\
\hline Forma de hojas & $\begin{array}{l}\text { ovadas a } \\
\text { elipticas }\end{array}$ & $\begin{array}{l}\text { elipticas a } \\
\text { lanceoladas }\end{array}$ & $\begin{array}{l}\text { ampliamente ovadas } \\
\text { a elipticas }\end{array}$ \\
\hline Largo de hojas & $(2-) 4.5-6.5(-9) \mathrm{cm}$ & $4-7 \mathrm{~cm}$ & $8-15 \mathrm{~cm}$ \\
\hline Ancho de hojas & $(1.5-) 2-3(-5.5) \mathrm{cm}$ & $1.5-2.5(-3) \mathrm{cm}$ & $6-9 \mathrm{~cm}$ \\
\hline $\begin{array}{l}\text { Corteza en rami- } \\
\text { llas de más de } \\
\text { un año }\end{array}$ & $\begin{array}{l}\text { persistente en } \\
\text { placas engrosadas, } \\
\text { rara vez exfoliante }\end{array}$ & $\begin{array}{l}\text { exfoliante o per- } \\
\text { sistente en placas } \\
\text { delgadas. }\end{array}$ & $\begin{array}{l}\text { persistente en } \\
\text { placas engrosadas }\end{array}$ \\
\hline
\end{tabular}

A diferencia de $A$. arizonica y $A$. tessellata, $A$. madrensis no se ve favorecida por el disturbio, aparentemente debido a sus mayores requerimientos de humedad y a la escasa tolerancia a la insolación durante su desarrollo. 
A. madrensis es un elemento común en bosques de pino-encino y bosques de pino en la Sierra Madre Occidental en Durango, en cañadas y laderas o mesas semihúmedas entre los 2400 y $2860 \mathrm{~m}$ de altitud. Los extremos de altitud registrados para la especie en otras áreas son de 2050 y $3000 \mathrm{~m}$. Las especies con las que se asocia con mayor frecuencia son Pinus cooperi, $P$. durangensis, $P$. leiophylla, Quercus sideroxyla, $Q$. rugosa y $Q$. crassifolia. Ocasionalmente se combina con Alnus y Abies en sitios húmedos, y con Pinus engelmannii, Quercus laeta y $Q$. eduardii en bosques semisecos.

La floración se presenta en marzo y abril, ocasionalmente desde enero, y los frutos maduran entre junio y agosto.

\section{AGRADECIMIENTOS}

Agradecemos cumplidamente a los encargados de los herbarios ENCB, IEB, MEXU y NMU el préstamo de sus colecciones de Arbutus, al Dr. Paul Sorensen la revisión en campo de algunas poblaciones del nuevo taxon, al Sr. Marco Antonio Ibarra García su esmerada elaboración del dibujo y a dos revisores anónimos las sugerencias para mejorar el manuscrito.

\section{LITERATURA CITADA}

González-Elizondo, S. y M. González-Elizondo. El género Arbutus (Ericaceae) en la Sierra Madre Occidental. Consideraciones sobre su taxonomía y distribución. Memorias de la Reunión Conmemorativa del XXX Aniversario del Herbario IBUG. Instituto de Botánica de la Universidad de Guadalajara. Guadalajara, Jalisco (en prensa).

González-Villarreal, L. M. 1990. Las Ericáceas de Jalisco, México. Colección Flora de Jalisco. Instituto de Botánica, Universidad de Guadalajara. Guadalajara, Jalisco. 140 pp.

McVaugh, R. \& T. J. Rosatti. 1978. A new species of Arbutus (Ericaceae) from western Mexico. Contr. Univ. Mich. Herb. 11: 301-304.

Small, J. K. 1914. Ericaceae. In: North Amer. Flora 29: 82-85.

Sorensen, P. D. 1987. Arbutus tessellata (Ericaceae), new from Mexico. Brittonia 39: 263-267.

Standley, P. C. 1924. Arbutus. In: Trees and shrubs of Mexico. Contr. U.S. Natl. Herb. 23: 1099-1100. 